\title{
Assistência fisioterapêutica na unidade de terapia intensiva à paciente com COVID- \\ 19: uma revisão integrativa
}

\author{
Physiotherapeutic care in the patient intensive care unit with COVID-19: an integrative review \\ Atención fisioterapéutica en la unidad de cuidados intensivos del paciente con COVID-19: una \\ revisión integradora
}




\title{
Lucas Sousa Guimarães \\ ORCID: https://orcid.org/0000-0002-9228-8114 Centro Universitário de Ciências e Tecnologia do Maranhão, Brasil E-mail: lucasguimaraes0310@gmail.com
}

\begin{abstract}
Resumo
A infecção COVID-19 causada pelo coronavírus (SARS-CoV-2) se alastrou tornando-se uma pandemia mundial levando a uma alta taxa de morbimortalidade. Os Fisioterapeutas, como profissionais que atuam na linha de frente da pandemia, devem estar sempre atentos às atualizações e ter habilidades para tomar decisões, realizar a detecção de novos casos e definir o tratamento adequado nas UTI. Objetivo: Analisar a assistência fisioterapêutica na Unidade de Terapia Intensiva à pacientes com COVID-19. Métodos: Trata-se de uma pesquisa bibliográfica do tipo revisão integrativa da literatura, na qual foi utilizado as bases de dados Bireme e PubMed, e os descritores em saúde e mesh terms para elaboração da estratégia de busca. Resultados: Foram incluídos nove estudos nesta revisão, estudos versaram sobre a assistência fisioterapêutica na UTI a pacientes com COVID-19. Destaca-se a experiência de fisioterapeutas e discutem os desafios e estratégia para o manejo clínico desses pacientes em UTIs de diferentes especialidades, como UTI cardiológica, neurológica, pediátrica, adulto etc. Conclusão: A assistência prestada deve focar em prevenir futuros agravos e fortalecer os pacientes de modo a melhorar a capacidade de troca de gases e oxigenação. Neste aspecto, o fisioterapeuta trabalha junto ao paciente buscando realizar atividades de prevenção e tratamento, além de oferecer apoio emocional, pois estes clientes, na maior parte dos casos, se encontram fragilizados física e psicologicamente.
\end{abstract}

Palavras-chave: Fisioterapeutas; COVID-19; Unidades de Terapia Intensiva.

\begin{abstract}
Introduction: The COVID-19 infection caused by the coronavirus (SARS-CoV-2) has spread to become a worldwide pandemic leading to a high rate of morbidity and mortality. Physiotherapists, as professionals who work on the front lines of the pandemic, must always be aware of updates and have the skills to make decisions, detect new cases and define the appropriate treatment in the ICU. Objective: To analyze physical therapy assistance in the Intensive Care Unit for patients with COVID-19. Methods: This is a bibliographic research of the integrative literature review type, in which the Bireme and PubMed databases, and the health descriptors and mesh terms were used to elaborate the search strategy. Results: Nine studies were included in this review, studies dealing with physical therapy assistance in the ICU for patients with COVID-19. The experience of physical therapists is highlighted and they discuss the challenges and strategy for the clinical management of these patients in ICUs of different specialties, such as cardiology, neurological, pediatric and adult ICUs. Conclusion: The care provided should focus on preventing future injuries and strengthening patients in order to improve their ability to exchange gas and oxygenation. In this aspect, the physiotherapist works with the patient, seeking to carry out prevention and treatment activities, in addition to offering emotional support, as these clients, in most cases, are physically and psychologically fragile.
\end{abstract}

Keywords: Physical therapists; COVID-19; Intensive Care Units.

\section{Resumen}

Introducción: La infección por COVID-19 causada por el coronavirus (SARS-CoV-2) se ha extendido hasta convertirse en una pandemia mundial que genera una alta tasa de morbilidad y mortalidad. Los fisioterapeutas, como profesionales que trabajan en primera línea de la pandemia, deben estar siempre al tanto de las actualizaciones y tener las habilidades para tomar decisiones, detectar nuevos casos y definir el tratamiento adecuado en la UCI. Objetivo: Analizar la asistencia de fisioterapia en la Unidad de Cuidados Intensivos para pacientes con COVID-19. Métodos: Se trata de una investigación bibliográfica del tipo revisión integradora de la literatura, en la que se utilizaron las bases de datos Bireme y PubMed, y los descriptores de salud y términos mesh para elaborar la estrategia de búsqueda. Resultados: Se incluyeron nueve estudios en esta revisión, estudios que tratan sobre la asistencia de fisioterapia en la UCI para pacientes con COVID-19. Se destaca la experiencia de los fisioterapeutas que discuten los retos y estrategia para el manejo clínico de estos pacientes en UCI de diferentes especialidades, como cardiología, neurológica, UCI pediátrica y de adultos. Conclusión: La atención brindada debe enfocarse en prevenir futuras lesiones y fortalecer a los pacientes para mejorar su capacidad de intercambio de gases y oxigenación. En este aspecto, el fisioterapeuta trabaja con el paciente, buscando realizar actividades de prevención y tratamiento, además de ofrecerle apoyo emocional, ya que estos clientes, en la mayoría de los casos, son física y psicológicamente frágiles.

Palabras clave: Fisioterapeutas; COVID-19; Unidades de Cuidados Intensivos.

\section{Introdução}

A Organização Mundial da Saúde (OMS) anunciou que a infecção COVID-19 causada pelo coronavírus (SARS-CoV-

2) se alastrou tornando-se uma pandemia mundial levando a uma alta taxa de morbimortalidade. A doença surgiu em seres 
humanos pela primeira vez na província de Huwan na China. Em relação aos principais sintomas estão: dispneia, febre, fadiga, tosse, secreções, cefaleia, aperto no peito e hemoptise. A incubação viral ocorre dentro de 2 a 11 dias, podendo ser transmitida tanto por pacientes assintomáticos, bem como sintomáticos (Cecchet et al., 2021).

A maioria dos pacientes infectados com a COVID-19 não chega a desenvolve complicações ou apresenta apenas sintomas relativamente leves, aproximadamente $14 \%$ evoluem para um estágio mais grave, em que requer hospitalização, suporte de oxigênio e, por vezes, ventilação mecânica (VM). Destes, de 5\% a 26\% dos casos necessitam internação em UTI. A COVID-19 pode desencadear complicações como sepse (59\%), lesão renal aguda (15\% a 29\%), disfunção cardíaca aguda (17\% a 23\%) e síndrome do desconforto respiratório agudo (SDRA) (31\% a 67\%) (Borges et al., 2020).

O Fisioterapeuta representa um papel de forma essencial em diversas partes do tratamento intensivo, como: no suporte aos indivíduos que necessita de um tratamento com apoio ventilatório; na prevenção de lesão por pressão com mobilização e mudanças de decúbito; nas abordagens pós-cirúrgicas; na avaliação e evolução do paciente; no controle e desmame ventilatório, sempre levando à extubação do alojado na Unidade de Terapia Intensiva (UTI); e, no manejo de técnicas respiratórias (Cecchet et al., 2021).

Os Fisioterapeutas, como profissionais que atuam na linha de frente da pandemia, devem estar sempre atentos às atualizações e ter habilidades para tomar decisões, realizar a detecção de novos casos e definir o tratamento adequado nas UTI. Junto com os coordenadores de serviço devem fornecer supervisão e suporte para auxiliar seus colaboradores no atendimento aos pacientes críticos, tendo sempre como base as diretrizes internacionais, nacionais, e/ou hospitalares direcionadas ao combate à COVID-19 (Musumeci et al., 2020). E exatamente por estarem na linha de frente, deve-se destacar que a pandemia de Covid-19 vem provocando o aparecimento de ansiedade, depressão, insônia, estresse e o agravamento de diversos transtornos psicológicos, principalmente a síndrome de Burnout, no qual indivíduos de várias classes profissionais passaram a apresentar (Melo et al., 2021; Da Silva et al., 2021).

Tendo por base estas reflexões, o presente estudo pretende responder a seguinte questão norteadora: Quais evidências mostram a assistência do fisioterapeuta na Unidade de Terapia Intensiva à pacientes com COVID-19? Visando sempre os fatos importantes como: as condutas usadas para o tratamento da mesma, e investigar os efeitos fisioterapêuticos no paciente com COVID-19 que se encontra no âmbito hospitalar.

O presente trabalho tem como objetivo geral: Analisar a assistência fisioterapêutica na Unidade de Terapia Intensiva à pacientes com COVID-19. E especificamente: destacar as principais atribuições dos fisioterapeutas na Unidade Terapia Intensiva (UTI); identificar sobre os principais fatores que interferem na qualidade da assistência fisioterapêutica durante a pandemia de Covid-19; e descrever sobre as estratégias e metodologias no âmbito da fisioterapia durante a pandemia de COVID-19.

\section{Metodologia}

O presente estudo trata-se de uma pesquisa bibliográfica do tipo revisão integrativa da literatura. Este procedimento foi escolhido por possibilitar a síntese e análise do conhecimento científico já produzido sobre o tema "Assistência fisioterapeutica na Unidade de Terapia Intensiva à paciente com COVID-19: uma revisão integrativa".

A metodologia da revisão integrativa da literatura dá suporte à PBE, que se encontra em desenvolvimento não só na enfermagem, como em todas as áreas de saúde. Considera-se pertinente abordar o conceito e as fases que constituem uma revisão integrativa da literatura, os instrumentos da PBE e a sua aplicabilidade na prática. Sendo essas fases: Identificação do tema e seleção da hipótese ou questão de pesquisa; Estabelecimento de critérios para inclusão e exclusão; Identificação dos estudos pré-selecionados e selecionados; Avaliação dos estudos incluídos; Intepretação dos resultados; e Apresentação da revisão/síntese do conhecimento (Sousa et al., 2017). 
O tema determinou a construção da estratégia PICO, que representa um acrônimo para Paciente ou Problema (P), Intervenção (I), Comparação (C) e Desfechos (O-outcomes), na qual foi utilizada para a geração da questão norteadora desta revisão integrativa da literatura: "Quais evidências mostram a assistência do fisioterapeuta na Unidade de Terapia Intensiva à pacientes com COVID-19?”.

Para a localização dos estudos relevantes, que respondessem à pergunta de pesquisa, utilizou-se de descritores indexados e não indexados (palavras-chave) nos idiomas português, inglês e espanhol. Os descritores foram obtidos a partir do Medical Subject Headings (MESH), e dos Descritores em Ciências da Saúde (DeCS).

Consultou-se por meio de descritores e palavras-chave as bases de dados PubMed da National Library of Medicine; e BVS (Biblioteca Virtual da Saúde), coordenada pela BIREME e composta de bases de dados bibliográficas produzidas pela Rede BVS, como LILACS, além da base de dados Medline e outros tipos de fontes, conforme demonstrado no Quadro 1.

Quadro 1. Elementos da estratégia PICO, descritores e palavras-chave utilizados. Caxias, MA, Brasil, 2021.

\begin{tabular}{|c|c|c|c|}
\hline \multicolumn{2}{|r|}{ Elementos } & Mesh & Decs \\
\hline $\mathrm{P}$ & $\begin{array}{l}\text { Pacientes com } \\
\text { Covid-19 }\end{array}$ & $\begin{array}{c}\text { COVID-19 } \\
\text { OR } \\
\text { SARS-CoV-2 } \\
\text { OR } \\
\text { "Coronavirus Infections" }\end{array}$ & $\begin{array}{c}\text { COVID-19 } \\
\text { OR } \\
\text { SARS-CoV-2 } \\
\text { OR } \\
\text { "Coronavirus Infections" }\end{array}$ \\
\hline $\mathrm{I}$ & $\begin{array}{c}\text { Assistência } \\
\text { fisioterapêutica }\end{array}$ & $\begin{array}{c}\text { "Physical Therapists" } \\
\text { OR } \\
\text { "Physical Therapy Department, } \\
\text { Hospital" } \\
\text { OR } \\
\text { Physical Therapy Specialty }\end{array}$ & $\begin{array}{c}\text { "Physical Therapists" } \\
\text { OR } \\
\text { "Physical Therapy Department, Hospital" } \\
\text { OR } \\
\text { Physical Therapy Specialty }\end{array}$ \\
\hline C & - & - & - \\
\hline $\mathrm{O}$ & Respiração & $\begin{array}{c}\text { "Intensive Care Units" } \\
\text { OR } \\
\text { Hospitals }\end{array}$ & $\begin{array}{c}\text { "Intensive Care Units" } \\
\text { OR } \\
\text { Hospitals }\end{array}$ \\
\hline
\end{tabular}

Fonte: Descritores e Palavras-chaves (2021).

O elemento C da estratégia PICO não foi abordado nesta pesquisa pois esta não tem por objetivo comparar intervenções. Os termos utilizados durante a pesquisa foram classificados e combinados nos bancos de dados, resultando em estratégias específicas de cada base, conforme demonstrado Quadro 2.

Quadro 2. Estratégias de busca utilizadas nas bases de dados BIREME e PUBMED. Caxias, MA, Brasil, 2021.

\begin{tabular}{|c|l|c|c|c|}
\hline Base dados & \multicolumn{1}{|c|}{ Estratégia de busca } & Resultados & Filtrados & Selecionados \\
\hline $\begin{array}{c}\text { BIREME } \\
\text { (Desc) }\end{array}$ & $\begin{array}{l}\text { (COVID-19 OR SARS-CoV-2 OR "Coronavirus Infections") AND } \\
\text { ("Physical Therapists" OR "Physical Therapy Department, } \\
\text { Hospital" OR "Physical Therapy Specialty") AND ("Intensive Care } \\
\text { Units" OR Hospitals) }\end{array}$ & 24 & 23 & 06 \\
\hline $\begin{array}{c}\text { PUBMED } \\
\text { (Mesh Terms) }\end{array}$ & $\begin{array}{l}\text { (COVID-19 OR SARS-CoV-2 OR "Coronavirus Infections") } \\
\text { AND ("Physical Therapists" OR "Physical Therapy Department, } \\
\text { Hospital" OR "Physical Therapy Specialty")) AND ("Intensive } \\
\text { Care Units" OR Hospitals) }\end{array}$ & 60 & 46 & 03 \\
\hline
\end{tabular}

Fonte: Bases de dados (2020).

Como critérios de inclusão utilizaram-se estudos disponíveis em sua totalidade, publicados nos últimos três anos, de 2019 até 2021, nos idiomas Português, Espanhol e Inglês. Foram excluídos da busca inicial capítulos de livros, resumos, textos incompletos, teses, dissertações, monografias, relatos técnicos e outras formas de publicação que não artigos científicos completos. A análise para seleção dos estudos foi realizada em duas fases, a saber: Na primeira, os estudos foram pré- 
selecionados segundo os critérios de inclusão e exclusão e de acordo com a estratégia de funcionamento e busca de cada base de dados.

Encontrou-se vinte e quatro (24) estudos como busca geral na BVS, sendo que limitando a busca para artigos com texto completo realizado com humanos nos últimos três anos, obteve-se vinte e três (23) estudos, destes foram analisados títulos e resumos onde apenas seis (06) estudos foram condizentes com a questão desta pesquisa.

$\mathrm{Na}$ base PUBMED, como busca total foram encontrados sessenta (60) estudos, aplicando na pesquisa o filtro que limita por texto completo dos últimos três anos com humanos, obteve-se quarenta e seis (46) estudos, destes foram analisados títulos e resumos e teve como resultado de três (03) estudos. Na segunda fase os estudos foram analisados quanto ao potencial de participação no estudo, avaliando o atendimento à questão de pesquisa, bem como o tipo de investigação, objetivos, amostra, método, resultados e conclusão, resultando em nove (09) artigos.

Posteriormente foram analisadas as informações coletadas nos artigos científicos e criadas categorias analíticas que facilitou a ordenação e a sumarização de cada estudo. Essa categorização foi realizada de forma descritiva, indicando os dados mais relevantes para o estudo. A pesquisa levou em consideração os aspectos éticos da pesquisa quanto às citações dos estudos, respeitando a autoria das ideias, os conceitos e as definições presentes nos artigos incluídos na revisão. Optou-se pela análise em forma estatística e de forma de texto, utilizando cálculos matemáticos e inferências, que serão apresentados em quadros e tabelas para facilitar a visualização e compreensão.

\section{Resultados}

Essa fase é organizada em duas partes. A primeira está relacionada com a caracterização dos estudos, já a segunda, relaciona-se ao cumprimento do objetivo do estudo, que consiste em conhecer a assistência fisioterapêutica na UTI a pacientes com COVID-19.

\section{Cacterização dos Estudos}

Foram incluídos nove estudos nesta revisão, a base de dados da Biblioteca Virtual em Saúde obteve maior número de evidências (66,6\%). A maioria dos estudos apresentaram abordagem qualitativa dos dados, correspondendo a sete $(77,8 \%)$ artigos. Com relação ao ano e idioma, a maioria das publicações concentraram-se no ano de 2020 (55,6\%); em que houve predomínio do idioma inglês (88,9\%). O Brasil foi o país com mais estudos incluídos (33,3\%). Quanto ao delineamento de pesquisa houve predomínio de estudos transversais, relatos de casos e de revisão, com cerca de $22,2 \%$ cada. O periódico Physical Therapy \& Rehabilitation Journal foi o que mais forneceu evidências (55,6\%), conforme demonstrado na Tabela 1. 
Tabela 1. Análise descritiva das produções científicas acerca da assistência fisioterapêutica na UTI a pacientes com COVID19. Caxias, MA, Brasil, 2021. (N=9).

\begin{tabular}{|c|c|c|}
\hline Variáveis & $\mathrm{N}$ & $\%$ \\
\hline \multicolumn{3}{|l|}{ Base de dados } \\
\hline BIREME & 06 & 66,6 \\
\hline PUBMED & 03 & 33,3 \\
\hline \multicolumn{3}{|l|}{ Abordagem do estudo } \\
\hline Quantitativa & 02 & 22,2 \\
\hline Qualitativa & 07 & 77,8 \\
\hline \multicolumn{3}{|l|}{ Ano } \\
\hline 2020 & 05 & 55,6 \\
\hline 2021 & 04 & 44,4 \\
\hline \multicolumn{3}{|l|}{ Idiomas } \\
\hline Português & 01 & 11,1 \\
\hline Inglês & 08 & 88,9 \\
\hline \multicolumn{3}{|l|}{ País } \\
\hline Brasil & 03 & 33,3 \\
\hline Suíça & 01 & 11,1 \\
\hline Colômbia & 01 & 11,1 \\
\hline China & 01 & 11,1 \\
\hline Argentina & 01 & 11,1 \\
\hline Estados Unidos & 01 & 11,1 \\
\hline Austrália & 01 & 11,1 \\
\hline \multicolumn{3}{|l|}{ Delineamento de pesquisa } \\
\hline Série de Casos & 01 & 11,1 \\
\hline Relato de Caso & 02 & 22,2 \\
\hline Estudo transversal & 02 & 22,2 \\
\hline Estudo de Revisão & 02 & 22,2 \\
\hline Estudo de Coorte & 01 & 11,1 \\
\hline Opinião de Especialistas & 01 & 11,1 \\
\hline \multicolumn{3}{|l|}{ Periódicos } \\
\hline Physical Therapy \& Rehabilitation Journal & 05 & 55,6 \\
\hline Clinics & 01 & 11,1 \\
\hline Revista Pesquisa em Fisioterapia & 01 & 11,1 \\
\hline Revista Brasileira de Terapia Intensiva & 01 & 11,1 \\
\hline Journal of Physiotherapy & 01 & 11,1 \\
\hline
\end{tabular}

Fonte: Bases de dados (2020).

O Quadro 3 mostra a distribuição dos estudos segundo número de ordem e base de dados, título, autores e anos de publicação, objetivo, perfil amostral e principais resultados. A maioria dos estudos versaram sobre a assistência fisioterapêutica na UTI a pacientes com COVID-19. Destaca-se a experiência de fisioterapeutas e discutem os desafios e estratégia para o manejo clínico desses pacientes em UTIs de diferentes especialidades, como UTI cardiológica, neurológica, pediátrica, adulto etc. As evidências fornecem protocolos assistenciais para o manejo clínico desses pacientes baseado na experiencia de diferentes profissionais especialistas de diversos países que vivenciaram e avaliaram essas intervenções na prática clínica, fornecendo subsídios para diversas instituições em todo o mundo. 
Research, Society and Development, v. 11, n. 1, e45411125021, 2022

(CC BY 4.0) | ISSN 2525-3409 | DOI: http://dx.doi.org/10.33448/rsd-v11i1.25021

Quadro 3. Publicações incluídas segundo o título do artigo, autor, objetivo principal, perfil amostral e principais resultados. Caxias, MA, Brasil. 2021. (N=09).

\begin{tabular}{|c|c|c|c|c|c|}
\hline $\begin{array}{c}\mathbf{N}^{0} \text { DE } \\
\text { ORDEM E } \\
\text { BASE }\end{array}$ & TÍTULO DO ARTIGO & $\begin{array}{l}\text { AUTOR/ } \\
\text { ANO }\end{array}$ & OBJETIVO PRINCIPAL & PERFIL AMOSTRAL & PRINCIPAIS RESULTADOS \\
\hline $\begin{array}{c}\text { A1 } \\
\text { BIREME }\end{array}$ & $\begin{array}{l}\text { Early Physical Therapist } \\
\text { Interventions for Patients With } \\
\text { COVID-19 in the Acute Care } \\
\text { Hospital: A Case Report Series }\end{array}$ & $\begin{array}{l}\text { Eggmann et } \\
\text { al. }(2021)\end{array}$ & $\begin{array}{l}\text { Descrever a experiência de } \\
\text { fisioterapeutas suíços no tratamento } \\
\text { de pacientes com COVID-19 durante } \\
\text { sua internação em cuidados agudos e } \\
\text { discutir os desafios e estratégias } \\
\text { potenciais no manejo clínico desses } \\
\text { pacientes. }\end{array}$ & $\begin{array}{l}11 \text { casos de pacientes com COVID- } \\
19 \text { de } 5 \text { hospitais suíços }\end{array}$ & $\begin{array}{l}\text { As intervenções variaram da educação do paciente ao posicionamento prono, } \\
\text { à mobilizção precoce e fisioterapia respiratória. Os pacientes ficavam } \\
\text { instáveis com rápida exacerbação dos sintomas e uma recuperação lenta e } \\
\text { flutuante. A maioria dos pacientes adotou as estratégias de tratamento } \\
\text { propostas, e a função pulmonar e a força física melhoraram com o tempo. }\end{array}$ \\
\hline $\begin{array}{c}\text { A2 } \\
\text { BIREME }\end{array}$ & $\begin{array}{l}\text { The First 60 Days: Physical } \\
\text { Therapy in a Neurosurgical Center } \\
\text { Converted Into a COVID-19 } \\
\text { Center in Brazil }\end{array}$ & $\begin{array}{l}\text { Gaspari et al. } \\
\text { (2020) }\end{array}$ & $\begin{array}{l}\text { Descrever a } \quad \text { atuação } r \text { dos } \\
\text { fisioterapeutas em um centro } \\
\text { neurocirúrgico que foi convertido em } \\
\text { centro COVID-19 para pacientes em } \\
\text { estado crítico. }\end{array}$ & $\begin{array}{l}\text { Um total de } 116 \text { casos confirmados } \\
\text { de COVID-19 foram tratados de } 17 \\
\text { de março a } 17 \text { de maio de } 2020 \text {. } \\
\text { Sessenta por cento eram homens } \\
\text { (70) e } 40 \% \text { eram mulheres (46), } \\
\text { com mediana de idade de } 59 \text { anos. }\end{array}$ & $\begin{array}{l}\text { O papel que o fisioterapeuta desempenhou no tratamento dos pacientes com } \\
\text { COVID-19 até o momento foi fundamental durante todo o período de } \\
\text { internação. A disponibilidade e experiência dos fisioterapeutas em UTI têm } \\
\text { facilitado o atendimento de eventos críticos, como intubação, posicionamento } \\
\text { do paciente, ajustes ventilatórios, extubação e treinamento funcional. }\end{array}$ \\
\hline $\begin{array}{c}\text { A3 } \\
\text { BIREME }\end{array}$ & $\begin{array}{l}\text { Translating COVID-19 Evidence } \\
\text { to Maximize Physical Therapists' } \\
\text { Impact and Public Health } \\
\text { Response }\end{array}$ & $\begin{array}{l}\text { Dean, Jones, } \\
\text { Yu, } \\
\text { Gosselink \& } \\
\text { Skinner } \\
(2020)\end{array}$ & $\begin{array}{l}\text { Descreve o COVID-19 como a } \\
\text { próxima virada histórica } \\
\text { crescimento e desenvolvimento da } \\
\text { profissão de fisioterapia }\end{array}$ & $\begin{array}{l}\text { Por meio de evidências científicas o } \\
\text { artigo retrata a COVID-19 e a } \\
\text { prática da fisioterapia na gestão da } \\
\text { UTI. }\end{array}$ & $\begin{array}{l}\text { Com relação ao tratamento contínuo para pacientes com COVID-19, os } \\
\text { fisioterapeutas precisam monitorar o estado cardiovascular e pulmonar e as } \\
\text { respostas às intervenções, seja mudando a posição do corpo na UTI ou } \\
\text { prescrevendo um programa de exercícios estruturado quando o paciente } \\
\text { retorna à comunidade. Os princípios e práticas de reabilitação pulmonar, } \\
\text { reabilitação cardíaca ou ambos devem ser aplicados conforme indicado com } \\
\text { base na avaliação inicial e na avaliação do paciente com COVID-19 e nas } \\
\text { respostas ao exercício. }\end{array}$ \\
\hline $\begin{array}{c}\text { A4 } \\
\text { BIREME }\end{array}$ & $\begin{array}{l}\text { Physical Therapist Management of } \\
\text { COVID-19 in the Intensive Care } \\
\text { Unit: The West China Hospital } \\
\text { Experience }\end{array}$ & $\begin{array}{l}\text { Li et al. } \\
(2021)\end{array}$ & $\begin{array}{l}\text { Relatar um serviço de fisioterapia } \\
\text { especialmente encomendado pela } \\
\text { Comissão de Saúde da Província de } \\
\text { Sichuan para gerenciar COVID-19 } \\
\text { durante a permanência de pacientes na } \\
\text { unidade de terapia intensiva (UTI) no } \\
\text { Centro Clínico de Saúde Pública de } \\
\text { Chengdu, na China. }\end{array}$ & $\begin{array}{l}\text { Uma coorte de } 16 \text { pacientes foi } \\
\text { admitida na UTI do Centro } \\
\text { Clínico de Saúde Pública de } \\
\text { Chengdu. }\end{array}$ & $\begin{array}{l}\text { Na alta da UTI, enquanto a maioria das medidas de desfecho foram quase } \\
\text { normais para a maioria dos pacientes, } 61 \% \text { e } 31 \% \text { desses pacientes tiveram } \\
\text { pico de fluxo expiratório e pressão inspiratória máxima, respectivamente, } \\
\text { abaixo de } 80 \% \text { do valor previsto e } 46 \% \text { apresentaram valores do Îndice de } \\
\text { Mobilidade De Morton abaixo do valor normativo. As funções respiratória e } \\
\text { física de alguns pacientes permaneceram ruins na alta da UTI, sugerindo que a } \\
\text { reabilitação em longo prazo pode ser necessária para esses pacientes. }\end{array}$ \\
\hline $\begin{array}{c}\text { A5 } \\
\text { BIREME }\end{array}$ & $\begin{array}{l}\text { Physiotherapy Care of Patients } \\
\text { with Coronavirus Disease } 2019 \\
\text { (COVID-19) - A Brazilian } \\
\text { Experience }\end{array}$ & $\begin{array}{l}\text { Righetti et } \\
\text { al. }(2020)\end{array}$ & $\begin{array}{l}\text { Descrever as diferentes ações e } \\
\text { práticas adotadas pelo Serviço de } \\
\text { Reabilitação do Hospital Sírio-Libanês } \\
\text { (São Paulo, Brasil) para enfrentar os } \\
\text { desafios de tratar e conter a } \\
\text { disseminação do COVID-19 }\end{array}$ & $\begin{array}{l}\text { Estudo realizado com base nas } \\
\text { opiniões de especialistas da linha } \\
\text { de frente e uma revisão da } \\
\text { literatura. }\end{array}$ & $\begin{array}{l}\text { A fisioterapia tem papel fundamental durante a internação do paciente. No } \\
\text { entanto, a equipe de fisioterapia do hospital deve ser bem orientada quanto } \\
\text { aos cuidados específicos para reduzir o risco de infecção e fornecer o melhor } \\
\text { atendimento ao paciente. }\end{array}$ \\
\hline $\begin{array}{c}\text { A6 } \\
\text { BIREME }\end{array}$ & $\begin{array}{l}\text { Intervenção fisioterapêutica em } \\
\text { uma criança com coronavírus em } \\
\text { um hospital de referência: relato } \\
\text { de caso }\end{array}$ & $\begin{array}{c}\text { Oliveira, } \\
\text { Veiga e } \\
\text { Mota (2021) }\end{array}$ & $\begin{array}{l}\text { Descrever o quadro clínico de uma } \\
\text { criança portadora de Cardiopatia } \\
\text { Congênita com Coronavírus positivo e } \\
\text { abordar a atuação fisioterapêutica } \\
\text { pediátrica. }\end{array}$ & $\begin{array}{l}\text { Relato de caso de uma paciente } \\
\text { de } 5 \text { anos e } 11 \text { meses de idade, } \\
\text { com diagnóstico antecedente de } \\
\text { Cardiopatia Congênita dos tipos: } \\
\text { Persistência do Canal Arterial e } \\
\text { Comunicação Interventricular. }\end{array}$ & $\begin{array}{l}\text { Os dados coletados por meio de análise de prontuário e observação clínica da } \\
\text { paciente demonstraram que a intervenção fisioterapêutica, quando contou com } \\
\text { técnicas de higiene brônquica, manobras reexpansivas e condutas motoras } \\
\text { ativas, contribuiu com a evolução clínica da paciente evidenciando melhora } \\
\text { na SpO2 e no desconforto ventilatório. }\end{array}$ \\
\hline
\end{tabular}


Research, Society and Development, v. 11, n. 1, e45411125021, 2022

(CC BY 4.0) | ISSN 2525-3409 | DOI: http://dx.doi.org/10.33448/rsd-v11i1.25021

\begin{tabular}{|c|c|c|c|c|c|}
\hline $\begin{array}{c}\text { A7 } \\
\text { PUBMED }\end{array}$ & $\begin{array}{l}\text { Description of physical } \\
\text { rehabilitation in intensive care } \\
\text { units in Argentina: usual practice } \\
\text { and during the COVID-19 } \\
\text { pandemic. Online survey }\end{array}$ & $\begin{array}{l}\text { Bertozzi, } \\
\text { Cagide, } \\
\text { Navarro \& } \\
\text { Accoce } \\
(2021)\end{array}$ & $\begin{array}{l}\text { Descrever a prática habitual de } \\
\text { assistência cinética motora e a unidade } \\
\text { de atenção intensiva de adultos, tanto } \\
\text { em pacientes com pecado COVID-19. }\end{array}$ & $\begin{array}{l}\text { Pesquisa online na qual } \\
\text { participaram fisioterapeutas que } \\
\text { trabalham em uma unidade de } \\
\text { terapia intensiva adulto na } \\
\text { Argentina. }\end{array}$ & $\begin{array}{l}\text { Dos } 351 \text { fisioterapeutas, } 76,1 \% \text { respondem que são os únicos responsáveis } \\
\text { pela mobilidade do paciente. A meta motora mais elevada variou de acordo } \\
\text { com quatro cenários de pacientes: pacientes ventilados mecanicamente, } \\
\text { pacientes desmamados da ventilação mecânica, pacientes que nunca } \\
\text { necessitaram de ventilação mecânica e pacientes com COVID-19 sob } \\
\text { ventilação mecânica. No primeiro e no último cenário, o maior objetivo era } \\
\text { otimizar a força muscular, enquanto nos outros dois, era realizar atividades da } \\
\text { vida diária. Por fim, a maior limitação em trabalhar com pacientes com } \\
\text { COVID-19 foi o isolamento respiratório e / ou de contato. }\end{array}$ \\
\hline $\begin{array}{c}\text { A8 } \\
\text { PUBMED }\end{array}$ & $\begin{array}{l}\text { Prone Positioning of Patients With } \\
\text { Acute Respiratory Distress } \\
\text { Syndrome Related to COVID-19: } \\
\text { A Rehabilitation-Based Prone } \\
\text { Team }\end{array}$ & $\begin{array}{l}\text { Ng et al. } \\
\text { (2020) }\end{array}$ & $\begin{array}{l}\text { Descrever a operação e evolução de } \\
\text { uma equipe prona composta por um } \\
\text { terapeuta de reabilitação. }\end{array}$ & $\begin{array}{lcr}\text { Equipe de } & \text { fisioterapeutas } \\
\text { terapeutas } & \text { ocupacionais com } \\
\text { experiência } & \text { em } \quad \text { cuidados } \\
\text { intensivos } & & \end{array}$ & $\begin{array}{l}\text { Vários fatores levaram ao estabelecimento bem-sucedido de uma equipe } \\
\text { propensa a reabilitação, incluindo conhecimento básico, treinamento, } \\
\text { colaboração multidisciplinar e avaliação de processo contínuo e } \\
\text { adaptabilidade. Ao longo de semanas fornecendo uma equipe voltada para a } \\
\text { reabilitação, o feedback verbal de várias equipes médicas foi extremamente } \\
\text { positivo. }\end{array}$ \\
\hline $\begin{array}{c}\text { A9 } \\
\text { PUBMED }\end{array}$ & $\begin{array}{l}\text { Physiotherapy management for } \\
\text { COVID-19 in the acute hospital } \\
\text { setting: clinical practice } \\
\text { recommendations }\end{array}$ & $\begin{array}{l}\text { Thomas et } \\
\text { al. (2020) }\end{array}$ & $\begin{array}{l}\text { Descrever recomendações para } \\
\text { gerenciamento de fisioterapia para } \\
\text { COVID-19 no ambiente hospitalar de } \\
\text { agudos. }\end{array}$ & $\begin{array}{l}\text { Um grupo de especialistas } \\
\text { internacionais em fisioterapia } \\
\text { cardiorrespiratória reuniu-se para } \\
\text { preparar rapidamente } \\
\text { recomendações clínicas para o } \\
\text { manejo fisioterapêutico do } \\
\text { COVID-19. }\end{array}$ & $\begin{array}{l}\text { Inclui: recomendações para o planejamento e preparação da força de trabalho } \\
\text { da fisioterapia; uma ferramenta de triagem para determinar a necessidade de } \\
\text { fisioterapia; e recomendações para a seleção de tratamentos fisioterapêuticos e } \\
\text { equipamentos de proteção individual. Destina-se ao uso por fisioterapeutas e } \\
\text { outras partes interessadas relevantes no ambiente de cuidados intensivos que } \\
\text { cuidam de pacientes adultos com COVID-19 confirmado ou suspeito. }\end{array}$ \\
\hline
\end{tabular}

Fonte: Bases de dados (2020). 


\section{Discussão}

\section{A assistência fisioterapêutica na unidade de terapia intensiva à pacientes com COVID-19}

No cenário da pandemia de Covid-19, muitas pessoas necessitaram de atendimento hospitalar, pois contraíram o vírus e desenvolveram sintomas mais graves da doença. Neste aspecto, o fisioterapeuta tem papel fundamental no tratamento dos pacientes tanto na ala clínica quanto na UTI, onde o profissional tem destaque dentro da UTI por facilitar o processo de realização de procedimentos em eventos críticos, como por exemplo, a intubação de pacientes (Gaspari et al., 2020). É visto que a fisioterapia visa restabelecer a força muscular e progressão para a realização de atividades de vida diária sem limitações ou com o mínimo de limitações possíveis, após o tempo de internação (Bertozzi et al., 2021).

É de extrema importância que os profissionais tenham um planejamento de gestão para nortear sua atuação no âmbito hospitalar, onde a gestão de fisioterapia voltada para o atendimento de pacientes com Covid-19 deve incluir: planejamento e preparação da força da equipe; ferramenta de triagem; e recomendações para a seleção de tratamentos de fisioterapia que deverão ser ofertados (Thomas et al., 2020). Corroborando com a afirmativa, Bertozzi et al. (2021) afirmam que, os fisioterapeutas devem estabelecer metas para aturem com pacientes acometidos por Covid-19 dentro da UTI e apontam que o isolamento é uma limitação para a mobilização dos pacientes.

Para tal, efetivar a assistência prestada é fundamental que os fisioterapeutas estejam atentos para quem realmente deve receber intervenções e quais seriam mais indicadas, neste ponto, é importante ressaltar que são indicadas ações em clientes com consolidação exsudativa, hipersecreção mucosa e/ou dificuldade em limpar secreções. E as principais intervenções são: mobilização e exercício, onde só serão realizadas ações mediante indicadores clínicos, evitando risco de contágio entre a equipe multiprofissional e desperdício de equipamentos de proteção individual (Thomas et al., 2020).

As principais intervenções realizadas pelos fisioterapeutas incluem: fisioterapia torácica convencional, exercício e mobilização precoce, instigando os clientes a se exercitarem para manter a funcionalidade; oxigenoterapia, tratamento de nebulização, ventilação não invasiva e oxigênio nasal de alto fluxo, para casos com gravidade leve a intermediária; intubação endotraqueal, ventilação mecânica protetora, e gerenciamento de ventilação mecânica em casos graves e refratários de hipoxemia. Pode ser indicado ainda, posicionamento prono, pressão do punho, tubo e suç̧ão nasotraqueal, uso de umidificador para pacientes ventilados, e métodos de desmamar pacientes ventilados e extubação (Righetti et al., 2020).

Em sintonia com a afirmativa, o estudo de Li et al. (2020) afirmam que a fisioterapia se concentra na prevenção da retenção de escarro, maximização da potência das vias aéreas, força respiratória e muscular dos membros e mobilidade funcional, onde por se tratar de uma patologia nova e que ainda precisa ser amplamente investigada, deve-se buscar a utilização de protocolos para padronizar a assistência e minimizar riscos para os pacientes e profissionais.

Intervenções precoces também merecem destaque, geralmente incluindo posicionamento prono, mobilização precoce e terapia respiratória, onde os procedimentos devem ser discutidos com a equipe para avaliar risco e benefícios. Trabalhar com pacientes internados por conta da Covid-19 é um desafio à fisioterapia, muitas vezes, esses indivíduos não tem capacidade para realizar exercícios por conta dos sintomas graves como fraqueza, disfagia pós-extubação, falha no desmame ou apresentarem ansiedade ou delírio. Os profissionais devem ser proativos e buscar alternativas terapêuticas especializadas e individualizadas que possam auxiliar a melhora na função pulmonar e a força física com o tempo (Eggmann et al., 2021).

Por fim, há diversas evidências cientificas que indicam a fisioterapia para tratar pacientes com Covid-19, porém, é notado que nos países desenvolvidos os fisioterapeutas não desenvolvem tantas ações dentro da UTI, já que o foco é a prevenção de sintomas graves, maximizando a troca de gases e condicionamento físico, reduzindo a frequência de necessidade de procedimentos invasivos e uso de medicamentos (Dean et al., 2020; Ng et al., 2020). 


\section{Principais fatores que interferem na qualidade da assistência fisioterapêutica durante a pandemia de COVID-19}

Diversos fatores podem interferir na assistência prestada pelo profissional fisioterapeuta no decorrer da pandemia, entre eles está o medo de contaminação, já que a Covid-19 possui um quadro complexo e de fácil transmissibilidade, o que torna o trabalho fisioterapêutico mais difícil, pois ele acontece por meio de intervenções geradoras de aerossol e as diretrizes internacionais sugeriam uma interferência mínima, exceto quando fosse indicado o contrário (Li et al., 2021).

O isolamento dos pacientes também tornou o processo de trabalho mais complicado (Eggmann et al., 2021). Corroborando com essa afirmação, estudo feito na Argentina com 351 fisioterapeutas que trabalham em UTIs de adultos indicou o isolamento respiratório e/ou de contato como a principal limitação enfrentada, exigindo maior atenção e dedicação dos trabalhadores (Bertozzi et al., 2021).

Segundo Eggmann et al. (2021), a própria realidade enfrentada e o fato de lidar com uma doença nova com poucas informações já representa um grande desafio. A grande instabilidade da oxigenação, a dispneia, ansiedade e o rápido descondicionamento de pacientes hospitalizados com Covid-19 fez com que alguns exercícios executados pelos fisioterapeutas necessitassem de adaptação individual, além de monitoramento constante da oxigenação.

Os estudos citaram ainda a falta de equipamentos de proteção individual, principalmente no começo da pandemia, como um aspecto que interfere na qualidade da assistência fisioterapêutica (Bertozzi et al., 2021). Semelhante a isso, Gaspari et al. (2020) afirma que no início, o Brasil se encontrava extremamente atrasado no que se refere a preparativos para Covid-19, os hospitais apresentavam escassez de EPI's; superlotação de leitos de UTI; e falta de testes virais, que eram realizados apenas em pacientes já em situação grave.

Dessa forma, todos esses aspectos causavam insegurança nos trabalhadores e tornavam as grandes jornadas de trabalho excessivamente cansativas, desgastando-os fisicamente e psicologicamente, fazendo até mesmo com que muitos se afastassem dos seus empregos.

\section{Estratégias e metodologias no âmbito da fisioterapia durante a pandemia de COVID-19}

Durante os meses iniciais de pandemia, os ambientes de a saúde passaram por modificações para assegurar a assistência aos inúmeros casos da doença, para adaptação dos profissionais de fisioterapia e demais área foram necessário estratégias como por exemplo, a contratação emergencial para aumentar os integrantes das equipes multidisciplinar e para fornecer apoio aos profissionais da linha de frente da COVID-19 (Gaspari et al. 2021; Dean et al. 2020).

Além disso o alto risco de contaminação e os medos associados a doença, levaram a elaboração de planos e produção de pesquisas na área, contribuindo na disseminação de evidências científicas com intuito de apresentar intervenções e medidas de cuidados aos profissionais (Li et al.; Dean et al., 2020).

Segundo Gaspari et al. (2021) os protocolos foram uma importante ferramenta adotada pelos profissionais de fisioterapia pois norteavam a prática e os procedimentos a serem realizados, bem como o papel do fisioterapeuta no processo de assistência a COVID-19. Os protocolos davam ênfase em pré-atendimento a pacientes com COVID-19, técnica de posicionamento, e principalmente gestão da ventilação, além da paramentação no ambiente hospitalar e em UTI (Dean et al., 2020).

Nesta perspectiva Thomas et al. (2020) afirmam que as recomendações foram fundamentais durante o período pandêmico, e que tinham como foco abordar sobre a adaptação do ambiente hospitalar, triagens, intervenções, proteção individual, sendo vista assim como escopo para a prática e atendimento de fisioterapia no contexto em questão. Entretanto além dessas metodologias, os treinamentos e capacitação das equipes multidisciplinar e dos fisioterapeutas dentre as exigências de assistência durante a pandemia foram considerados de extrema importância pelos profissionais, pois forneceram uma preparação nos aspectos físicos e psicológicos para lidar com a doença, e organizar o fluxo de atendimento e estabelecer 
respostar rápidas e alternativas para lidar com os pacientes críticos (Gaspari et al., 2021).

\section{Considerações Finais}

Este estudo permitiu observar a assistência prestada pelos fisioterapeutas dentro da unidade de terapia intensiva aos pacientes acometidos por Covid-19, destacando as principais intervenções realizadas e as principais atribuições desses profissionais. Foi evidenciado ainda, os fatores que interferem na qualidade da assistência e o preparo dos profissionais para atuarem frente à pandemia dentro da UTI.

Diante do exposto, torna-se claro que a assistência prestada deve focar em prevenir futuros agravos e fortalecer os pacientes de modo a melhorar a capacidade de troca de gases e oxigenação dos mesmos. Neste aspecto, o fisioterapeuta trabalha junto ao paciente buscando realizar atividades de prevenção e tratamento, além de oferecer apoio emocional, pois estes clientes, na maior parte dos casos, se encontram fragilizados física e psicologicamente.

Já que a fisioterapia exerce importante papel para a reabilitação dos pacientes internados na UTI devido ao acometimento por Covid-19, e conecta os clientes e demais profissionais, é importante que o fisioterapeuta avalie a assistência prestada e trabalhe em conjunto com a equipe multiprofissional. Deve-se destacar ainda, as principais intervenções realizadas com os clientes, sendo elas: posicionamento prono, mobilização precoce e terapia respiratória, sempre de acordo com a necessidade de cada paciente e seguindo protocolos de padronização para efetivar o cuidado.

Como limitações do estudo têm-se o fato da doença ser nova e ainda não ter sua fisiopatologia totalmente esclarecida, neste sentido, as pesquisas existentes precisam ser revisadas ou aprimoradas no futuro. Além disso, não há muita literatura disponível sobre as melhores intervenções para tratar os pacientes com Covid-19.

Por fim, o estudo possibilitou expandir os conhecimentos acerca da pandemia de Covid-19, e a atuação do fisioterapeuta dentro da UTI junto a pacientes afetados por Covid-19. Dessa forma, considera-se que novas pesquisas devem ser realizadas nesta linha de investigação, a fim de provar a importância das intervenções e assistência de fisioterapia.

\section{Referências}

Bertozzi, M. N., Cagide, S., Navarro, E., \& Accoce, M. (2021). Description of physical rehabilitation in intensive care units in Argentina: usual practice and during the covid-19 pandemic. online survey. Revista Brasileira de Terapia Intensiva, 33(2), 188-195.

Borges, D. L., Rapello, G. V. G., Deponti, G. N., \& Andrade, F. M. D. D. (2020). Posição prona no tratamento da insuficiência respiratória aguda na COVID19. Assobrafir Ciência, 11(1), 111-120.

Cecchet, I. L., De Lima, M. C., De Souza, I. F. (2021). Fisioterapia respiratória no tratamento hospitalar da covid-19: uma revisão integrativa. Revista Artigos. Com, 26, 6242-6242.

Da Silva, W. C., Da Silva, C. O., Melo, K. C.., Soares, A. N., Hernandes, L. F., Araújo, Z. A. M., \& Sousa, F. D. C. A. (2021). Explorando os impactos na saúde mental de crianças durante a pandemia de covid-19. International Journal of Development Research, 11(04), 46248-46253.

Dean, E., Jones, A., Yu, H. P. M., Gosselink, R., \& Skinner, M. (2020). Translating COVID-19 Evidence to Maximize Physical Therapists' Impact and Public Health Response. Physical Therapy, 100(9), 1458-1464.

Eggmann, S., Kindler, A., Perren, A., Ott, N., Johannes, F., Vollenweider, R., \& Jakob, S. M. (2020). Early Physical Therapist Interventions for Patients With COVID-19 in the Acute Care Hospital: a case report series. Physical Therapy, 101(1), 1-9.

Gaspari, C. H., Assumpção, I., Freire, R., Silva, A., Santiso, C., \& Jaccoud, A. C. (2020). The First 60 Days: physical therapy in a neurosurgical center converted into a covid-19 center in brazil. Physical Therapy, 100(12), 2120-2126.

Li, L., Yu, P., Yang, M., Xie, W., Huang, L., He, C., \& Jones, A. Y. M. (2020). Physical Therapist Management of COVID-19 in the Intensive Care Unit: the west china hospital experience. Physical Therapy, 101(1), 1-10.

Melo, K. C., Da Silva, C. O., Souza, M. W. S. S., Soares, A. N., Da Silva, W. C., Oliveira, F. B. M., ... \& Da Silva, R. A. (2021). Síndrome de burnout em profissionais da saúde que atuam na linha de frente da covid-19. International Journal of Development Research, 11(04), 44706-44711.

Musumeci, M. M., Martinez, B. P., Nogueira, I. C., \& Alcanfor, T. (2020). Recursos fisioterapêuticos utilizados em unidades de terapia intensiva para avaliação e tratamento das disfunções respiratórias de pacientes com covid-19. Assobrafir Ciência, 11(1), 73-86. 
Research, Society and Development, v. 11, n. 1, e45411125021, 2022

(CC BY 4.0) | ISSN 2525-3409 | DOI: http://dx.doi.org/10.33448/rsd-v11i1.25021

Ng, J A., Miccile, L., Iracheta, C., Berndt, C., Detwiller, M., Yuse, C., \& Tolland, J. (2020). Prone Positioning of Patients With Acute Respiratory Distress Syndrome Related to COVID-19: a rehabilitation-based prone team. Physical Therapy, 100(10), 1737-1745.

Oliveira, J. S. D., Veiga, I. N., \& Mota, C. S. (2021). Intervenção fisioterapêutica em uma criança com coronavírus em um hospital de referência: relato de caso. Revista Pesquisa em Fisioterapia, 11(1), 227-232.

Righetti, R. F., Onoue, M. A., Politi, F. V. A., Teixeira, D. T., Souza, P. N. D., Kondo, C. S., \& Pastore, L. (2020). Physiotherapy Care of Patients with Coronavirus Disease 2019 (COVID-19) - A Brazilian Experience. Clinics, 75.

Sousa, L. M. M., Marques-Vieira, C. M. A, Severino, S. S. P., \& Antunes, A. V. (2017). A metodologia de revisão integrativa da literatura em enfermagem. Revista de Investigação de Enfermagem, 21, 17-26.

Thomas, P., Baldwin, C., Bissett, B., Boden, I., Gosselink, R., Granger, C. L., \& Moses, R. (2020). Physiotherapy management for COVID-19 in the acute hospital setting: clinical practice recommendations. Journal Of Physiotherapy, 66(2), 73-82. 